\title{
Universal torsors and values of quadratic polynomials represented by norms
}

\author{
Ulrich Derenthal · Arne Smeets · Dasheng Wei
}

Received: 24 July 2012 / Revised: 30 May 2014 / Published online: 27 September 2014

(C) Springer-Verlag Berlin Heidelberg 2014

\begin{abstract}
Let $K / k$ be an extension of number fields, and let $P(t)$ be a quadratic polynomial over $k$. Let $X$ be the affine variety defined by $P(t)=N_{K / k}(\mathbf{z})$. We study the Hasse principle and weak approximation for $X$ in three cases. For [K:k] $=4$ and $P(t)$ irreducible over $k$ and split in $K$, we prove the Hasse principle and weak approximation. For $k=\mathbb{Q}$ with arbitrary $K$, we show that the BrauerManin obstruction to the Hasse principle and weak approximation is the only one. For $[K: k]=4$ and $P(t)$ irreducible over $k$, we determine the Brauer group of smooth proper models of $X$. In a case where it is non-trivial, we exhibit a counterexample to weak approximation.
\end{abstract}

Mathematics Subject Classification $14 \mathrm{G} 05$ (11D57, 14F22)

U. Derenthal $(\varangle)$

Institut für Algebra, Zahlentheorie und Diskrete Mathematik, Leibniz Universität Hannover, Welfengarten 1, 30167 Hannover, Germany

e-mail: derenthal@math.uni-hannover.de

A. Smeets

Departement Wiskunde, KU Leuven, Celestijnenlaan 200B, 3001 Leuven, Belgium

A. Smeets

Département de Mathématiques, Université Paris-Sud 11, Bâtiment 425, 91405 Orsay, France e-mail: arnesmeets@gmail.com

D. Wei

Academy of Mathematics and System Science, CAS, Beijing 100190, People's Republic of China e-mail: dshwei@amss.ac.cn 


\section{Introduction}

Let $K / k$ be an extension of number fields of degree $n$. When can values of a polynomial $P(t)$ over $k$ be represented by norms of elements of $K$ ? To answer this natural question, we study solutions $(t, \mathbf{z}) \in k \times K$ of the equation

$$
P(t)=N_{K / k}(\mathbf{z}) \text {. }
$$

This question is closely related to the study of the Hasse principle and weak approximation (see the end of this introduction for a review of this terminology) on a smooth proper model $X^{c}$ of the affine hypersurface $X \subset \mathbb{A}_{k}^{1} \times \mathbb{A}_{k}^{n}$ with coordinates $(t, \mathbf{z})=\left(t, z_{1}, \ldots, z_{n}\right)$ defined by (1), via a choice of a basis $\omega_{1}, \ldots, \omega_{n}$ of $K$ over $k$, with $N_{K / k}(\mathbf{z})=N_{K / k}\left(z_{1} \omega_{1}+\cdots+z_{n} \omega_{n}\right)$.

Colliot-Thélène conjectured that the Brauer-Manin obstruction to weak approximation is the only one on $X^{c}$ (see [3]). This conjecture is known in the case where $P(t)$ is constant, thanks to work of Sansuc [19]; if additionally $K / k$ is cyclic, it is known that the Hasse principle (proved by Hasse himself [15, p. 150]) and weak approximation hold. Other known cases of Colliot-Thélène's conjecture, in some cases leading to a proof of the Hasse principle and weak approximation, include the class of Châtelet surfaces $([K: k]=2$ and $\operatorname{deg}(P(t)) \leq 4)[8,9]$, a class of singular cubic hypersurfaces ([K:k] $=3$ and $\operatorname{deg}(P(t)) \leq 3)$ [5] and the case where $K / k$ is arbitrary and $P(t)$ is split over $k$ with at most two distinct roots [4,16,21]. Finally, if one admits Schinzel's hypothesis, then the conjecture is known for $K / k$ cyclic and $P(t)$ arbitrary $[10,12]$. See for example [3, Introduction] and [1, Section 1] for a more detailed discussion of these results and the difficulties of this problem.

The obvious next challenge is the case where $P(t)$ is an irreducible quadratic polynomial. Browning and Heath-Brown recently obtained a positive result in this direction: they proved the Hasse principle and weak approximation for $[K: k]=4$ and $\operatorname{deg}(P(t))=2$ with $P(t)$ irreducible over $k$ and split in $K$, with $k=\mathbb{Q}$. Their main result [1, Theorem 1] therefore answers a question raised in [4, Section 2] positively in the case $k=\mathbb{Q}$. In this paper, we extend their work in several directions, using a variety of different techniques.

We give a very short proof of this result for an arbitrary number field $k$. It is independent of the work of Browning and Heath-Brown and gives a simple geometric proof of their result. More specifically, we show that an open subset of $X$ is a smooth fibration in 3-dimensional quadrics defined by quadratic forms of full rank over a conic. The result follows by applying the theorem of Hasse-Minkowski to the base and to the fiber.

Theorem 1 Let $P(t)$ be a quadratic polynomial that is irreducible over a number field $k$ and split in $K$ with $[K: k]=4$. Then the Hasse principle and weak approximation hold for the variety $X \subset \mathbb{A}_{k}^{5}$ defined by (1).

If the ground field is $\mathbb{Q}$, we can prove a much more general result based on the analytic work of Browning and Heath-Brown in [1, Theorem 2] and the descent method of Colliot-Thélène and Sansuc: 
Theorem 2 Let $k=\mathbb{Q}$ and $K$ be any number field. Let $P(t) \in \mathbb{Q}[t]$ be an arbitrary quadratic polynomial. Then the Brauer-Manin obstruction to the Hasse principle and weak approximation is the only obstruction on any smooth proper model of $X \subset \mathbb{A}_{\mathbb{Q}}^{n+1}$ defined by (1).

Let $X$ be the variety defined by Eq. (1) and let $U \subset X$ be the open subvariety given by $P(t) \neq 0$. We prove that the variety $Y$ defined by $[1,(1.5)]$ is the restriction $\mathcal{T}_{U}$ of a universal torsor $\mathcal{T}$ over $X$ to $U$, or a product of $\mathcal{T}_{U}$ with a quasi-split torus. For the variety $Y$, [1, Theorem 2] proves weak approximation using sieve methods from analytic number theory, inspired by work of Fouvry and Iwaniec [13]. While one step in Browning's and Heath-Brown's deduction of [1, Theorem 1] from [1, Theorem 2] leads to their restriction to $[K: \mathbb{Q}]=4$, the combination of their analytic work with descent theory gives our more general Theorem 2 . We also generalize Theorem 2 to a large class of multivariate polynomials $P\left(t_{1}, \ldots, t_{\ell}\right) \in \mathbb{Q}\left[t_{1}, \ldots, t_{\ell}\right]$.

Note that the descent argument in our proof of Theorem 2 follows the proof of [16, Theorem 2.2]. As pointed out by the referee, the more sophisticated method of [4] would allow the treatment of smooth proper models of $X \subset \mathbb{A}_{\mathbb{Q}}^{n+1}$ defined by the more general equation

$$
c P(t)^{n}=N_{K / \mathbb{Q}}(\mathbf{z})
$$

for any $n \geq 1$, with $c \in \mathbb{Q}^{\times}$, quadratic $P(t) \in \mathbb{Q}[t]$ and any number field $K$. See also [24].

Our Theorem 2 then leads naturally to the question in which cases there may be a Brauer-Manin obstruction. We give a complete analysis for $[K: k]=4$ and $P(t)$ irreducible over $k=\mathbb{Q}$. If $P(t)$ is split in $K$, there is no Brauer-Manin obstruction to weak approximation by [1, Theorem 1] or our Theorem 1 , so that it is not very surprising that the Brauer group of a smooth proper model of $X$ is trivial in this case. Otherwise, the Brauer group is sometimes non-trivial:

Theorem 3 Let $P(t)$ be a quadratic polynomial that is irreducible over a number field $k$ and has splitting field $L$, and let $[K: k]=4$. Let $X^{c}$ be a smooth proper model of $X \subset \mathbb{A}_{k}^{5}$ defined by (1).

The Brauer group $\operatorname{Br}\left(X^{c}\right)$ contains non-constant elements if and only if the extension $K / k$ is not Galois, $P(t)$ is not split in $K$ and the extension $K \cdot L / k$ is Galois with $\operatorname{Gal}(K \cdot L / L) \cong \mathbb{Z} / 4 \mathbb{Z}$.

In Sect. 5, we prove a more detailed version of Theorem 3 that includes an explicit description of the Brauer group in the non-trivial case (Theorem 4). The calculations are quite involved and it seems unlikely that similar detailed results can be obtained for higher degree extensions.

As a corollary, we get the validity of the Hasse principle and weak approximation in some new cases when the Brauer group is trivial (Corollary 3). In Sect. 6, we use our explicit description of the Brauer group in the case where it is non-trivial to produce a counterexample to weak approximation (explained by the Brauer-Manin obstruction).

Terminology For an algebraic variety $Z$ defined over a number field $k$ with algebraic closure $\bar{k}$, one says that the Hasse principle holds if $\prod_{v \in \Omega_{k}} Z\left(k_{v}\right) \neq \varnothing$ (where $\Omega_{k}$ 
is the set of places of $k$ and $k_{v}$ is the completion of $k$ at $\left.v\right)$ implies $Z(k) \neq \emptyset$. One says that weak approximation holds if $Z(k)$ is dense in $\prod_{v \in \Omega_{k}} Z\left(k_{v}\right)$ with the product topology, via the diagonal embedding.

If $Z$ is smooth and proper, one says that the Brauer-Manin obstruction to the Hasse principle is the only one if $\left(\prod_{v \in \Omega_{k}} Z\left(k_{v}\right)\right)^{\operatorname{Br}(Z)} \neq \varnothing$ implies that $Z(k) \neq \varnothing$, and that the Brauer-Manin obstruction to weak approximation is the only one if $Z(k)$ is dense in $\left(\prod_{v \in \Omega_{k}} Z\left(k_{v}\right)\right)^{\operatorname{Br}(Z)}$. Here $\left(\prod_{v \in \Omega_{k}} Z\left(k_{v}\right)\right)^{\operatorname{Br}(Z)}$ is the set of all $\left(z_{v}\right) \in$ $\prod_{v \in \Omega_{k}} Z\left(k_{v}\right)$ satisfying $\sum_{v \in \Omega_{k}} \operatorname{inv}_{v}\left(A\left(z_{v}\right)\right)=0$ for each $A$ in the Brauer group $\operatorname{Br}(Z)=H_{\text {ét }}^{2}\left(Z, \mathbb{G}_{\mathrm{m}}\right)$ of $Z$, where the map $\operatorname{inv}_{v}: \operatorname{Br}\left(k_{v}\right) \rightarrow \mathbb{Q} / \mathbb{Z}$ is the invariant map from local class field theory.

The subgroup $\operatorname{Br}_{0}(Z)$ of constant elements in the Brauer group is the image of the natural map $\operatorname{Br}(k) \rightarrow \operatorname{Br}(Z)$. The algebraic Brauer group $\operatorname{Br}_{1}(Z)$ is the kernel of the natural map $\operatorname{Br}(Z) \rightarrow \operatorname{Br}(\bar{Z})$, where $\bar{Z}=Z \times{ }_{k} \bar{k}$.

\section{Quadratic polynomials represented by a quartic norm}

In this section, we give a very short proof of Theorem 1 that is independent of the work of Browning and Heath-Brown [1] and generalizes it from $\mathbb{Q}$ to an arbitrary number field $k$.

Proof of Theorem 1 Using a change of variables if necessary, we can assume that the irreducible quadratic polynomial $P(t) \in k[t]$ that is split in $K$ (with $[K: k]=4$ ) has the form $P(t)=c\left(t^{2}-a\right)$, with $c \in k^{\times}$, where $a \in k^{\times}$is not a square and $\sqrt{a} \in K$. Write $L=k(\sqrt{a}) \subset K$.

Let $U=\{(t, \mathbf{z}): P(t) \neq 0\} \subset X$. Let $S \subset \mathbb{A}_{k}^{2}$ be the conic defined by the affine equation $N_{L / k}(\mathbf{w})=c$ and let $p: U \rightarrow S$ be the morphism defined by

$$
(t, \mathbf{z}) \mapsto(t-\sqrt{a})^{-1} N_{K / L}(\mathbf{z}) .
$$

Theorem 1 holds by [8, Proposition 3.9], which is based on the theorem of HasseMinkowski, since this is a smooth fibration in quadrics of dimension 3 over a base satisfying weak approximation.

Indeed, the base $S$ is a conic, where weak approximation holds, and the statement regarding the fibers can be checked over an algebraic closure $\bar{k}$ of $k$. Here, we have

$\bar{U}=U \times_{k} \bar{k} \cong\left\{c\left(t^{2}-a\right)=u_{1} u_{2} u_{3} u_{4}\right\} \subset \mathbb{A}_{\bar{k}}, \bar{S}=S \times_{k} \bar{k} \cong\left\{w_{1} w_{2}=c\right\} \subset \mathbb{A}_{\bar{k}}^{2}$,

with $p$ mapping $\left(t, u_{1}, \ldots, u_{4}\right) \in \bar{U}$ to $\left((t-\sqrt{a})^{-1} u_{1} u_{2},(t+\sqrt{a})^{-1} u_{3} u_{4}\right) \in \bar{S}$. Hence the fiber over $\left(w_{1}, w_{2}\right) \in \bar{S}$ is

$$
\left\{t-\sqrt{a}=w_{1}^{-1} u_{1} u_{2}, t+\sqrt{a}=w_{2}^{-1} u_{3} u_{4}\right\} \subset \mathbb{A}_{k} \frac{5}{k}
$$

Eliminating $t$ gives

$$
\left\{-2 \sqrt{a}=w_{1}^{-1} u_{1} u_{2}-w_{2}^{-1} u_{3} u_{4}\right\} \subset \mathbb{A} \frac{4}{k},
$$


where the quadratic form in $u_{1}, \ldots, u_{4}$ clearly has rank 4 . Hence the fiber is a smooth quadric of dimension 3.

Remark 1 The analog of Theorem 1 holds for global fields of positive characteristic different from 2 as well. Indeed, it is not hard to see that our arguments and the proof of [8, Proposition 3.9] remain valid for such fields.

\section{Universal torsors}

The basic strategy to prove Theorem 2 is based on the following result, which reduces the problem of the Hasse principle and weak approximation on a variety to the same questions on its universal torsors, where we have no Brauer-Manin obstructions. This kind of result has been proved essentially by Colliot-Thélène and Sansuc in their seminal paper [6]. However, they developed their theory under the simplifying assumption that the varieties involved are proper. Skorobogatov developed a variant under less stringent assumptions in [22]. Descent on open varieties also features in [11] and [3]. We will use the following variant:

Proposition 1 Let $Z$ be a smooth, geometrically rational variety over a number field $k$ with algebraic closure $\bar{k}$. Let $\bar{Z}=Z \times{ }_{k} \bar{k}$. Assume furthermore that $\bar{k}[Z]^{\times}=\bar{k}^{\times}$, that $\operatorname{Pic}(\bar{Z})$ is free of finite rank, that universal $Z$-torsors exist and that any universal $Z$-torsor satisfies weak approximation. Then the Brauer-Manin obstruction to the Hasse principle and weak approximation is the only one for any smooth proper model $Z^{c}$ of $Z$.

The condition $\bar{k}[Z]^{\times}=\bar{k}^{\times}$means that the only invertible regular functions on $Z$ are the constant ones. The proof of this proposition is straightforward; the key observation is the fact that $Z\left(\mathbb{A}_{k}\right)^{\mathrm{Br} 1(Z)}$ is dense in $\left(\prod_{v} Z^{c}\left(k_{v}\right)\right)^{\mathrm{Br}\left(Z^{c}\right)}$ by [11, Proposition 1.1] (note that $\operatorname{Br}\left(\overline{Z^{c}}\right)=0$ ), and the result then follows from descent theory and the implicit function theorem.

The main result of this section is concerned with the existence of universal torsors [6, (2.0.4)] over $X$ as in (1) and their local description.

Let us first recall some more definitions. If $k$ is a field and if $A$ is an étale $k$-algebra, then the $k$-variety $R_{A / k}\left(\mathbb{G}_{\mathrm{m}, A}\right)$ is defined via its functor of points: take $R_{A / k}\left(\mathbb{G}_{\mathrm{m}, A}\right)(B)=\left(A \otimes_{k} B\right)^{\times}$functorially for every $k$-algebra $B$. The norm map $N_{A / k}$ is defined as in $[2, \S 12.2]$. We denote the absolute Galois group of $k$ by $\Gamma_{k}$.

Proposition 2 Let $K / k$ be an extension offields of degree $n$. Let $P(t)$ be an irreducible separable polynomial of degree $r$ over $k$.

The variety $X \subset \mathbb{A}_{k}^{n+1}$ defined by (1) is smooth and geometrically integral, with $\operatorname{Pic}(\bar{X})$ free of finite rank and $\bar{k}[X]^{\times}=\bar{k}^{\times}$. Let $U$ be the open subset of $X$ defined by $P(t) \neq 0$. Then $\operatorname{Pic}(\bar{U})=0$.

Let $c \in k^{\times}$be the leading coefficient of $P(t)$, let $L$ be the field $k[t] /(P(t))$ and let $\eta$ be the class of $t$ in $L$. Let $A=L \otimes_{k} K$. For any universal torsor $\mathcal{T}$ over $X$, there exists a solution $(\rho, \xi) \in L^{\times} \times K^{\times}$of the equation

$$
c N_{L / k}(\rho)=N_{K / k}(\xi)
$$


such that $\mathcal{T}_{U}$ (its restriction to $U$ ) is isomorphic to the subvariety of $\mathbb{A}_{k}^{1} \times R_{A / k}\left(\mathbb{G}_{\mathrm{m}, A}\right)$ (with coordinates $(t, \mathbf{z})$ ) given by the equation

$$
t-\eta=\rho N_{A / L}(\mathbf{z}) \text {. }
$$

Conversely, for any solution $(\rho, \xi) \in L^{\times} \times K^{\times}$of (2), there is a universal torsor $\mathcal{T}$ over $X$ such that $\mathcal{T}_{U}$ has such a description.

Using only the basic definitions, it is easy to see that one can specialize Eq. (3) as follows in the two "extreme" cases:

(a) If $P(t)$ splits completely in $K$, then $\mathcal{T}_{U}$ is isomorphic to the sub-variety of $\mathbb{A}_{k}^{1} \times$ $\left(R_{K / k}\left(\mathbb{G}_{\mathrm{m}, K}\right)\right)^{r}$ (with coordinates $\left.\left(t, \mathbf{x}_{1}, \ldots, \mathbf{x}_{r}\right)\right)$ given by the equation

$$
t-\eta=\rho \prod_{i=1}^{r} \sigma_{i}^{-1}\left(N_{K / \sigma_{i}(L)}\left(\mathbf{x}_{i}\right)\right)
$$

where $\sigma_{1}, \ldots, \sigma_{r}$ is a set of representatives of $\Gamma_{k} / \Gamma_{L}$.

(b) If $P(t)$ remains irreducible in $K$, then $\mathcal{T}_{U}$ is isomorphic to the subvariety of $\mathbb{A}_{k}^{1} \times$ $R_{F / k}\left(\mathbb{G}_{\mathrm{m}, F}\right)$ (with coordinates $\left.(t, \mathbf{x})\right)$ given by the equation

$$
t-\eta=\rho N_{F / L}(\mathbf{x})
$$

where $F=L \cdot K$.

The proof of Proposition 2 is an adaptation of [16, Theorem 2.2] and will occupy most of the remainder of this section. The $\bar{k}$-variety $\bar{X}$ can be described by an equation of the form

$$
c \prod_{i=1}^{r}\left(t-\eta_{i}\right)=u_{1} \cdots u_{n}
$$

where $\eta_{1}, \ldots, \eta_{r}$ are the embeddings of $\eta$ in $\bar{k}$. We note that $X$ is smooth because $P(t)$ is separable. Consider the morphism $p: X \rightarrow \mathbb{A}_{k}^{1}$ given by $(t, \mathbf{x}) \mapsto t$. Over $\bar{k}$, it has precisely $r$ reducible fibers $X_{i}$, for $i=1, \ldots, r$, over $t=\eta_{i}$. Each of these has $n$ irreducible components $D_{i, j}=\left\{t=\eta_{i}, u_{j}=0\right\}$ for $j=1, \ldots, n$. Let $U_{0}$ be the open subset of $\mathbb{A}_{k}^{1}$ where $P(t) \neq 0$ and let $U=p^{-1}\left(U_{0}\right) \subset X$. We have

$$
\bar{U}=U \times_{k} \bar{k} \cong\left(\mathbb{A}_{\bar{k}} \backslash\left\{\eta_{1}, \ldots, \eta_{r}\right\}\right) \times \mathbb{G}_{\mathrm{m}, \bar{k}}^{n-1},
$$

so that $\operatorname{Pic}(\bar{U})=0$.

We have $\bar{k}[X]^{\times}=\bar{k}^{\times}$. Indeed, the generic fiber of $\bar{X} \rightarrow \mathbb{A}_{\frac{k}{1}}$ is $\mathbb{G}_{\mathrm{m}, \bar{k}(t)}^{n-1}$. Therefore, any $f \in \bar{k}[X]^{\times}$has the form $f=g(t) u_{1}^{m_{1}} \cdots u_{n}^{m_{n}}$ with $g \in k(t)$ and $m_{1}, \ldots, m_{n} \in \mathbb{Z}$. 
If $g(t)$ has a root or pole in some $t_{0} \notin\left\{\eta_{1}, \ldots, \eta_{r}\right\}$, then $f$ or $f^{-1}$ is not regular in a point on $p^{-1}\left(t_{0}\right)$. Otherwise, we have

$$
g(t)=c^{\prime} \prod_{i=1}^{r}\left(t-\eta_{i}\right)^{e_{i}}
$$

for some $c^{\prime} \in \bar{k}^{\times}$and $e_{1}, \ldots, e_{r} \in \mathbb{Z}$. Then

$$
\operatorname{div}(f)=\sum_{i=1}^{r} \sum_{j=1}^{n}\left(e_{i}+m_{j}\right) D_{i, j},
$$

so $f \in \bar{k}[X]^{\times}$if and only if $e_{1}=\cdots=e_{r}=-m_{1}=\cdots=-m_{n}$. By (6), this is equivalent to saying that $f$ is a constant in $\bar{k}^{\times}$.

By descent theory [6, Corollary 2.3.4], universal torsors over $X$ exist if and only if the exact sequence of $\Gamma_{k}$-modules

$$
1 \rightarrow \bar{k}^{\times} \rightarrow \bar{k}[U]^{\times} \rightarrow \bar{k}[U]^{\times} / \bar{k}^{\times} \rightarrow 1
$$

is split.

It is easy to see that the abelian group $\bar{k}[U]^{\times} / \bar{k}^{\times}$is free of rank $r+n-1$, generated by the classes of the functions $t-\eta_{1}, \ldots, t-\eta_{r}, u_{1}, \ldots, u_{n}$ with an obvious $\Gamma_{k}$-action and the relation

$$
\sum_{i=1}^{r}\left[t-\eta_{i}\right]-\sum_{j=1}^{n}\left[u_{j}\right]=0
$$

because of the equation defining $X$.

The exact sequence (7) is split if and only if the classes can be lifted to $\bar{k}[U]^{\times}$in a $\Gamma_{k}$-equivariant way, via a map

$$
\phi: \bar{k}[U]^{\times} / \bar{k}^{\times} \rightarrow \bar{k}[U]^{\times}, \quad[t-\eta] \mapsto \rho^{-1}(t-\eta), \quad\left[u_{1}\right] \mapsto \xi^{-1} u_{1}
$$

where $\rho \in L^{\times}$and $\xi \in K^{\times}$. Because of the unique relation (8), the pair $(\rho, \xi) \in$ $L^{\times} \times K^{\times}$defines such a splitting if and only if it satisfies (2).

We now want to apply [6, Theorem 2.3.1, Corollary 2.3.4] for the local description of universal torsors over $X$. We will describe a morphism of tori $d: M \rightarrow T$ such that its dual map of characters fits into the following commutative diagram of $\Gamma_{k}$-equivariant homomorphisms.

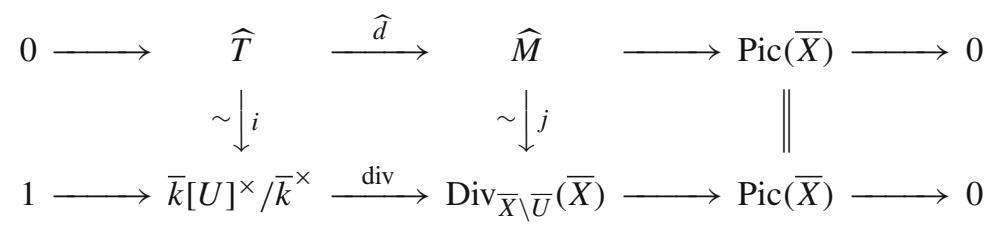


Here, the second row is exact because $\operatorname{Pic}(\bar{U})=0$ and $\bar{k}[X]^{\times}=\bar{k}^{\times}$.

The $\Gamma_{k}$-module $\bar{k}[U]^{\times} / \bar{k}^{\times}$is isomorphic to the module of characters of the algebraic $k$-torus $T \subset R_{L / k}\left(\mathbb{G}_{\mathrm{m}, L}\right) \times R_{K / k}\left(\mathbb{G}_{\mathrm{m}, K}\right)$ with coordinates $\left(\mathbf{z}_{1}, \mathbf{z}_{2}\right)$ given by

$$
N_{L / k}\left(\mathbf{z}_{1}\right)=N_{K / k}\left(\mathbf{z}_{2}\right) \text {. }
$$

Indeed, the character group $\widehat{T}$ is the quotient of $\mathbb{Z}\left[\Gamma_{k} / \Gamma_{L}\right] \oplus \mathbb{Z}\left[\Gamma_{k} / \Gamma_{K}\right]$ with the diagonal $\Gamma_{k}$-action by the relation

$$
\sum_{\sigma \Gamma_{L} \in \Gamma_{k} / \Gamma_{L}} \sigma \Gamma_{L}=\sum_{\gamma \Gamma_{K} \in \Gamma_{k} / \Gamma_{K}} \gamma \Gamma_{K}
$$

The isomorphism $i: \widehat{T} \rightarrow \bar{k}[U]^{\times} / \bar{k}^{\times}$is given by

$$
i\left(\sigma \Gamma_{L}\right)=[t-\sigma(\eta)], \quad i\left(\gamma \Gamma_{K}\right)=\left[\gamma\left(u_{1}\right)\right]
$$

The abelian group $\operatorname{Div}_{\bar{X} \backslash \bar{U}}(\bar{X})$ is free of rank $r n$, generated by $D_{i, j}$ for $i=1, \ldots, r$ and $j=1, \ldots, n$. There is a bijection $\Gamma_{k} / \Gamma_{L} \times \Gamma_{k} / \Gamma_{K} \rightarrow\left\{D_{i, j}\right\}$ defined by $\left(\sigma \Gamma_{L}, \gamma \Gamma_{K}\right) \mapsto\left\{t=\sigma(\eta), \gamma\left(u_{1}\right)=0\right\}$ that is compatible with the action of $\Gamma_{k}$, acting diagonally on the left hand side. Recalling $A=L \otimes_{k} K$, this shows that $\operatorname{Div}_{\bar{X} \backslash \bar{U}}(\bar{X})$ is isomorphic to the module of characters of the $k$-torus $M=R_{A / k}\left(\mathbb{G}_{\mathrm{m}, A}\right)$. Let $j: \widehat{M} \rightarrow \operatorname{Div}_{\bar{X} \backslash \bar{U}}(\bar{X})$ be this isomorphism.

Consider the homomorphism div $: \bar{k}[U]^{\times} / \bar{k}^{\times} \rightarrow \operatorname{Div}_{\bar{X} \backslash \bar{U}}(\bar{X})$ that maps a function to its divisor. We have

$$
\operatorname{div}([t-\eta])=\sum_{j=1}^{n} D_{1, j}, \quad \operatorname{div}\left(\left[u_{1}\right]\right)=\sum_{i=1}^{r} D_{i, 1} .
$$

Therefore, the abelian group $\operatorname{Pic}(\bar{X})$ is free of rank $(r-1)(n-1)$, with a basis consisting of the classes $\left[D_{i, j}\right]$ for $i=1, \ldots, r-1$ and $j=1, \ldots, n-1$. Now div induces a homomorphism on the character modules $\widehat{d}: \widehat{T} \rightarrow \widehat{M}$. The dual of this homomorphism is then given by the morphism of $k$-tori

$$
d: M \rightarrow T, \quad \mathbf{z} \mapsto\left(N_{A / L}(\mathbf{z}), N_{A / K}(\mathbf{z})\right)
$$

Let $S$ be the Néron-Severi torus dual to the $\Gamma_{k}$-module $\operatorname{Pic}(\bar{X})$, so that we have an exact sequence of tori

$$
1 \rightarrow S \rightarrow M \rightarrow T \rightarrow 1
$$

This makes $M$ into a $T$-torsor under $S$.

We now describe the map $U \rightarrow T$ induced by the splitting $\phi$ as in (9) by a choice of $(\rho, \xi) \in L^{\times} \times K^{\times}$satisfying (2). The induced map is given by

$$
U \rightarrow T, \quad(t, \mathbf{x}) \mapsto\left(\rho^{-1}(t-\eta), \xi^{-1} \mathbf{x}\right),
$$


and it is easy to see that the image is in $T$ using the equation of $X$ and the condition (2). Therefore, the image of $U$ in $T$ is isomorphic to the subvariety of $\mathbb{A}_{k}^{1} \times T$ with coordinates $\left(t, \mathbf{z}_{1}, \mathbf{z}_{2}\right)$ defined by

$$
t-\eta=\rho \mathbf{z}_{1}
$$

By [6, Theorem 2.3.1, Corollary 2.3.4], any universal torsor $\mathcal{T}_{U}$ over $U$ is the pullback of a torsor $M$ from $T$ to $U$. Our computations show that it is isomorphic to the subvariety of $\mathbb{A}_{k}^{1} \times R_{A / k}\left(\mathbb{G}_{\mathrm{m}, A}\right)$ with coordinates $(t, \mathbf{z})$ defined by (4). This completes the proof of Proposition 2.

Remark 2 One can determine equations for universal torsors $\mathcal{T}$ over the smooth locus $X_{\mathrm{sm}}$ of the variety $X$ defined by (1) even if $P(t)$ is not irreducible over $k$; note that $X$ is not smooth if $P(t)$ is not separable. Then $\operatorname{Pic}\left(X_{\mathrm{sm}}\right)$ is a finitely generated (but not necessarily free) abelian group. So $\mathcal{T}$ will be a torsor over $X_{\text {sm }}$ under the group of multiplicative type that is dual to $\operatorname{Pic}\left(X_{\mathrm{sm}}\right)$.

The result is as follows: assume that

$$
P(t)=c P_{1}(t)^{e_{1}} \cdots P_{d}(t)^{e_{d}}
$$

for $c \in k^{\times}$, some irreducible monic polynomials $P_{i}(t) \in k[t]$ and positive integers $e_{i}$. Write $L_{i}=k[t] /\left(P_{i}(t)\right)$ and let $\eta_{i}$ be the class of $t$ in $L_{i}$. For $i=1, \ldots, d$, consider the étale $L_{i}$-algebra $A_{i}=L_{i} \otimes_{k} K$. Let $U \subset X_{\mathrm{sm}}$ be the open subvariety given by $P(t) \neq 0$. For any universal torsor $\mathcal{T}$ over $X_{\mathrm{sm}}$, there exists a solution $\left(\rho_{1}, \ldots, \rho_{d}, \xi\right) \in L_{1}^{\times} \times \cdots \times L_{d}^{\times} \times K^{\times}$of the equation

$$
c N_{L_{1} / k}\left(\rho_{1}\right)^{e_{1}} \cdots N_{L_{d} / k}\left(\rho_{d}\right)^{e_{d}}=N_{K / k}(\xi)
$$

such that $\mathcal{T}_{U}$ is isomorphic to the subvariety of $\mathbb{A}_{k}^{1} \times \prod_{i=1}^{d} R_{A_{i} / k}\left(\mathbb{G}_{\mathrm{m}, A_{i}}\right)$ with coordinates $\left(t, \mathbf{z}_{1}, \ldots, \mathbf{z}_{d}\right)$ given by the system of equations

$$
t-\eta_{i}=\rho_{i} N_{A_{i} / L_{i}}\left(\mathbf{z}_{i}\right) \quad \text { for } 1 \leq i \leq d .
$$

Conversely, for any solution of (10), there is a universal torsor $\mathcal{T}$ over $X_{\mathrm{sm}}$ with such a local description.

Note that [16, Theorem 2.2] is a special case of this result. The proof is an adaptation of the proof of [16, Theorem 2.2] or Proposition 2.

In case that $k$ is a number field, we can link the existence of universal torsors as in Proposition 2 to the absence of Brauer-Manin obstructions on $X$. We have the following general statement, suggested to us by the referee:

Lemma 1 Let $X$ be a smooth, geometrically integral variety over a number field $k$ such that $\bar{k}[X]^{\times}=\bar{k}^{\times}$. Assume that $\operatorname{Pic}(\bar{X})$ is a finitely generated abelian group. If there is no Brauer-Manin obstruction to the Hasse principle on a smooth proper model of $X$, then universal torsors over $X$ exist. 
Proof Since $\operatorname{Pic}(\bar{X})$ is finitely generated, there exists an open subset $U \subset X$ such that $\operatorname{Pic}(\bar{U})=0$. By [6, Proposition 2.2.8], universal $X$-torsors exist if and only if the exact sequence

$$
1 \rightarrow \bar{k}^{\times} \rightarrow \bar{k}[U]^{\times} \rightarrow \bar{k}[U]^{\times} / \bar{k}^{\times} \rightarrow 1
$$

of Galois modules is split. The same result applies, of course, to any smooth proper model $X^{c}$ of $X$. Hence universal $X$-torsors exist if and only if universal $X^{c}$-torsors exist; by [23, Proposition 6.1.4], this is the case when $X^{c}\left(\mathbb{A}_{k}\right)^{\operatorname{Br}\left(X^{c}\right)} \neq \emptyset$.

\section{Quadratic polynomials represented by a norm over $\mathbb{Q}$}

Let $k=\mathbb{Q}$. As before, we can assume without loss of generality that $P(t)=c\left(t^{2}-a\right)$ with $c \in \mathbb{Q}^{\times}$and $a \in \mathbb{Q}$, but now we do not assume that $P(t)$ is split in $K$. Using the deep work of Browning and Heath-Brown and our description of universal torsors, we can prove the following result:

Proposition 3 If the quadratic polynomial $P(t)$ is irreducible over $\mathbb{Q}$, then each universal torsor over $X \subset \mathbb{A}_{k}^{n+1}$ defined by (1) satisfies weak approximation.

Proof Let $\mathcal{T}$ be a universal torsor over $X$, with $\mathcal{T}_{U}$ as in Proposition 2. It is enough to prove weak approximation on $\mathcal{T}_{U}$.

Assume that $P(t)$ is split in $K$. Consider $\mathcal{T}_{U} \subset \mathbb{A}_{k}^{1} \times\left(R_{K / k}\left(\mathbb{G}_{\mathrm{m}, K}\right)\right)^{2}$ defined by Eq. (4) in the case $r=2$. Let $L=k(\sqrt{a})$. For any $\sigma \in \Gamma_{k}$, we have $\sigma(L)=L$, and for any $x \in L$, we have $\sigma(x)=\sigma^{-1}(x)$. Therefore, (4) can be rewritten as

$$
t-\sqrt{a}=\rho N_{K / L}\left(\mathbf{x}_{1}\right) \cdot \sigma\left(N_{K / L}\left(\mathbf{x}_{2}\right)\right)
$$

where $\sigma \in \Gamma_{k}$ with $\sigma(\sqrt{a})=-\sqrt{a}$.

The variety given by this equation is isomorphic to the subvariety $Y$ of $\mathbb{A}_{k}^{1} \times$ $\left(R_{K / k}\left(\mathbb{G}_{\mathrm{m}, K}\right)\right)^{2}$ defined by the equation

$$
N_{K / k}(\mathbf{w})(t-\sqrt{a})=\rho N_{K / L}(\mathbf{y}),
$$

via the substitution

$$
\mathbf{w}=\mathbf{x}_{2}^{-1}, \quad \mathbf{y}=\mathbf{x}_{1} \mathbf{x}_{2}^{-1}
$$

with inverse

$$
\mathbf{x}_{1}=\mathbf{w}^{-1} \mathbf{y}, \quad \mathbf{x}_{2}=\mathbf{w}^{-1}
$$

using $N_{K / k}\left(\mathbf{x}_{2}\right)=N_{L / k}\left(N_{K / L}\left(\mathbf{x}_{2}\right)\right)=N_{K / L}\left(\mathbf{x}_{2}\right) \cdot \sigma\left(N_{K / L}\left(\mathbf{x}_{2}\right)\right)$. This is exactly [1, equation (1.5)]. Weak approximation then holds on $Y$ because of [1, Theorem 2]. 
Assume now that $P(t)$ remains irreducible over $K$ and write $F=K \cdot L$, where $L=k(\sqrt{a})$. Choose some $\sigma \in \Gamma_{K}$ such that $\sigma \notin \Gamma_{F}=\Gamma_{L} \cap \Gamma_{K}$, so $\sigma \notin \Gamma_{L}$. Therefore, $\sigma$ is a representative of the non-trivial class both in $\Gamma_{K} / \Gamma_{F}$ and in $\Gamma_{k} / \Gamma_{L}$.

Let $\gamma_{1}, \ldots, \gamma_{n}$ be a set of coset representatives of $\Gamma_{L} / \Gamma_{F}$. We claim that a set of representatives of $\Gamma_{k} / \Gamma_{F}$ is given by $\gamma_{1}, \ldots, \gamma_{n}, \gamma_{1} \sigma, \ldots, \gamma_{n} \sigma$. Indeed, if $\gamma_{i} \sigma \Gamma_{F}=$ $\gamma_{j} \sigma \Gamma_{F}$, then we have $\sigma^{-1} \gamma_{j}^{-1} \gamma_{i} \sigma \in \Gamma_{F}=\Gamma_{L} \cap \Gamma_{K}$. Using $\sigma \in \Gamma_{K}$, this gives $\gamma_{j}^{-1} \gamma_{i} \in \sigma \Gamma_{K} \sigma^{-1}=\Gamma_{K}$, and we have $\gamma_{j}^{-1} \gamma_{i} \in \Gamma_{L}$ by definition. Hence $\gamma_{j}^{-1} \gamma_{i} \in$ $\Gamma_{L} \cap \Gamma_{K}=\Gamma_{F}$, so $\gamma_{i} \Gamma_{F}=\gamma_{j} \Gamma_{F}$, which implies $i=j$. Furthermore, if $\gamma_{i} \sigma \Gamma_{F}=$ $\gamma_{j} \Gamma_{F}$, then $\gamma_{j}^{-1} \gamma_{i} \sigma \in \Gamma_{F} \subset \Gamma_{L}$, which contradicts the fact that $\gamma_{i}, \gamma_{j} \in \Gamma_{L}$, but $\sigma \notin \Gamma_{L}$. Finally, $\gamma_{i} \Gamma_{F}=\gamma_{j} \Gamma_{F}$ only for $i=j$ by construction. This proves the claim.

Therefore, $N_{F / k}(\mathbf{w})=N_{F / L}(\mathbf{w}) N_{F / L}(\sigma(\mathbf{w}))$. We note that $\sigma$ induces a $k$ automorphism of the variety $R_{F / k}\left(\mathbb{G}_{\mathrm{m}, F}\right)$ : this is clear from the functor-of-points description of $R_{F / k}\left(\mathbb{G}_{\mathrm{m}, F}\right)$.

Using this observation, we see that the variety $Y^{\prime} \subset \mathbb{A}_{k}^{1} \times\left(R_{F / k}\left(\mathbb{G}_{\mathrm{m}, F}\right)\right)^{2}$ with coordinates $(t, \mathbf{w}, \mathbf{y})$ defined by

$$
N_{F / k}(\mathbf{w})(t-\sqrt{a})=\rho N_{F / L}(\mathbf{y})
$$

(i.e. Eq. (11) with $K$ replaced by $F)$ is isomorphic to the product $\mathcal{T}_{U} \times R_{F / k}\left(\mathbb{G}_{\mathrm{m}, F}\right)$ with coordinates $(t, \mathbf{x}, \mathbf{y})$ subject to (5). The isomorphism is defined by the map

$$
(t, \mathbf{w}, \mathbf{y}) \mapsto\left(t,(\mathbf{w} \sigma(\mathbf{w}))^{-1} \mathbf{y}, \mathbf{w}\right)
$$

the inverse substitution being given by

$$
(t, \mathbf{x}, \mathbf{y}) \mapsto(t, \mathbf{y}, \mathbf{x y} \sigma(\mathbf{y}))
$$

Since $Y^{\prime}$ satisfies weak approximation by [1, Theorem 2] and since $R_{F / k}\left(\mathbb{G}_{\mathrm{m}, F}\right)$ is rational and therefore has non-trivial $k_{v}$-points for any place $v$, this implies that $\mathcal{T}_{U}$ satisfies weak approximation.

Proof of Theorem 2 If $P(t)$ is split over $\mathbb{Q}$ with two distinct roots, then Theorem 2 is a special case of [16, Theorem 1.1]. If it is split over $\mathbb{Q}$ with one double root, $U \subset X$ as in Proposition 2 is a principal homogeneous space of a torus, and Theorem 2 holds by [19].

Next, assume that $P(t)$ is irreducible over $\mathbb{Q}$. Assume that there is no BrauerManin obstruction to the Hasse principle on a smooth and proper model of $X$. Then Lemma 1 shows that universal torsors $\mathcal{T}$ over $X$ exist. By Proposition $3, \mathcal{T}_{U}$ satisfies weak approximation. Proposition 2 shows that $\bar{k}[X]^{\times}=\bar{k}^{\times}$and that $\operatorname{Pic}(\bar{X})$ is free of finite rank. Then an application of Proposition 1 gives the result.

Corollary 1 If the quadratic polynomial $P(t) \in \mathbb{Q}[t]$ is not split in the Galois closure of $K / \mathbb{Q}$, then the Hasse principle and weak approximation hold on any smooth proper model of $X \subset \mathbb{A}_{\mathbb{Q}}^{n+1}$ defined by (1). 
Proof By [25, Theorem 2.2], the smooth proper model $X^{c}$ satisfies $\operatorname{Br}\left(X^{c}\right)=$ $\mathrm{Br}_{0}\left(X^{c}\right)$, so the result follows immediately from Theorem 2 .

Finally, we generalize Theorem 2 to equations involving a multivariate polynomial $P\left(t_{1}, \ldots, t_{\ell}\right)$, using techniques developed by Harari in [14]:

Corollary 2 Let $P_{0}, P_{1}, P_{2}$ be polynomials in $\ell-1$ variables $t_{2}, \ldots, t_{\ell}$ over $\mathbb{Q}$ of arbitrary degree satisfying

$$
\operatorname{gcd}\left(P_{0}\left(t_{2}, \ldots, t_{\ell}\right), P_{1}\left(t_{2}, \ldots, t_{\ell}\right), P_{2}\left(t_{2}, \ldots, t_{\ell}\right)\right)=1
$$

Let $K$ be an arbitrary number field of degree $n=[K: \mathbb{Q}]$. Then the Brauer-Manin obstruction to the Hasse principle and weak approximation is the only obstruction on any smooth proper model of $X \subset \mathbb{A}_{\mathbb{Q}}^{n+\ell}$ defined by the equation

$$
t_{1}^{2} \cdot P_{2}\left(t_{2}, \ldots, t_{\ell}\right)+t_{1} \cdot P_{1}\left(t_{2}, \ldots, t_{\ell}\right)+P_{0}\left(t_{2}, \ldots, t_{\ell}\right)=N_{K / \mathbb{Q}}(\mathbf{z})
$$

Proof Consider the projection $\pi: X \rightarrow \mathbb{A}_{\mathbb{Q}}^{\ell-1}$ defined by $(\mathbf{t}, \mathbf{z}) \mapsto\left(t_{2}, \ldots, t_{\ell}\right)$ and consider the closed subset

$$
F=\left\{P_{0}\left(t_{2}, \ldots, t_{\ell}\right)=P_{1}\left(t_{2}, \ldots, t_{\ell}\right)=P_{2}\left(t_{2}, \ldots, t_{\ell}\right)=0\right\}
$$

of $\mathbb{A}_{\mathbb{Q}}^{\ell-1}$, which is of codimension at least 2 by assumption.

The fibers of $\pi$ over $\mathbb{A}_{\mathbb{Q}}^{\ell-1} \backslash F$ are geometrically integral. The fiber over each rational point in this set is defined by $P\left(t_{1}\right)=N_{K / \mathbb{Q}}(\mathbf{z})$ for some non-zero polynomial $P\left(t_{1}\right)$ of degree at most 2. By Theorem 2 for quadratic $P\left(t_{1}\right)$, by rationality for linear $P\left(t_{1}\right)$ and by [19] for constant $P\left(t_{1}\right)$, this has the property that the Brauer-Manin obstruction to the Hasse principle and weak approximation is the only obstruction on any smooth proper model. The generic fiber of $\pi$ is a rational variety. Therefore, the result follows by an application of [14, Théorème 3.2.1] (where we can check over $\bar{k}$ that condition $(*)$ of that result holds in our case).

\section{Brauer groups for quartic norms}

For $X$ defined by (1) with $K / k$ a quartic extension of number fields, we show in this section that the Brauer group of a smooth proper model $X^{c}$ of $X$ is trivial in certain cases. Then an application of Theorem 2 proves the Hasse principle and weak approximation on $X$ for $k=\mathbb{Q}$. In other cases, we show that the Brauer group is nontrivial. In the next section, we will also give an explicit example which illustrates the fact that a non-trivial Brauer class can give rise to an obstruction to weak approximation in this situation.

Recall that the Brauer group is a birational invariant of smooth, proper varieties: therefore it suffices to study the Brauer group of a given (smooth) compactification of $X$. Following the ideas developed in [4, Section 2], we will use a certain partial compactification $Y$ of $X$ contained in a smooth proper model $X^{c}$. It suffices to consider 
this particular model. Note that the natural maps $\operatorname{Br}\left(X^{c}\right) \rightarrow \operatorname{Br}(Y) \rightarrow \operatorname{Br}(X)$ are injective.

A classical argument, based on the Hochschild-Serre spectral sequence (see [4, Proposition 2.3]), gives an exact sequence of the form

$$
0 \rightarrow \operatorname{Br}_{0}(Y) \rightarrow \operatorname{Br}(Y) \rightarrow H^{1}(k, \operatorname{Pic}(\bar{Y})) \rightarrow H^{3}\left(k, \bar{k}^{\times}\right)
$$

If $k$ is a number field, then $H^{3}\left(k, \bar{k}^{\times}\right)=0$. Let $\Gamma_{k}$ be the abolute Galois group of $k$. Let $L$ be the splitting field of $P(t)$.

Lemma 2 Consider the norm one torus $T=R_{K / k}^{1}\left(\mathbb{G}_{\mathrm{m}, K}\right)$. Let $T^{c}$ be a smooth compactification of $T$ appearing in the construction of the partial compactification $Y$ of $X$ as in [4, Section 2]. Then

$$
0 \rightarrow Q_{L} / \operatorname{Res}_{k / L}\left(Q_{k}\right) \rightarrow H^{1}(k, \operatorname{Pic}(\bar{Y})) \rightarrow H^{1}\left(k, \operatorname{Pic}\left(\overline{T^{c}}\right)\right)
$$

is exact, where

$$
\begin{aligned}
Q_{k} & =\operatorname{ker}\left(H^{1}(k, \mathbb{Q} / \mathbb{Z}) \rightarrow H^{1}(K, \mathbb{Q} / \mathbb{Z})\right), \\
Q_{L} & =\operatorname{ker}\left(H^{1}(L, \mathbb{Q} / \mathbb{Z}) \rightarrow H^{1}\left(K \otimes_{k} L, \mathbb{Q} / \mathbb{Z}\right)\right) .
\end{aligned}
$$

Here, $\operatorname{Res}_{k / L}: H^{1}(k, \mathbb{Q} / \mathbb{Z}) \rightarrow H^{1}(L, \mathbb{Q} / \mathbb{Z})$ and all other maps are restriction maps.

Proof Because of [4, Proposition 2.5], we have the exact sequence

$$
0 \rightarrow H^{1}\left(k, \widehat{T} \otimes_{\mathbb{Z}} \mathbb{Z}\left[\Gamma_{k} / \Gamma_{L}\right]\right) / j_{*} H^{1}(k, \widehat{T}) \rightarrow H^{1}(k, \operatorname{Pic}(\bar{Y})) \rightarrow \amalg_{\omega}^{2}(\widehat{T})_{P} \rightarrow 0,
$$

where $j: \mathbb{Z} \rightarrow \mathbb{Z}\left[\Gamma_{k} / \Gamma_{L}\right]$ is defined by $1 \mapsto \sum_{\sigma \Gamma_{L} \in \Gamma_{k} / \Gamma_{L}} \sigma \Gamma_{L}$. Furthermore, $\widehat{T}$ is the character group of $T$ and $\amalg_{\omega}^{2}(\widehat{T})_{P}$ is a subgroup of $\amalg_{\omega}^{2}(\widehat{T})$ [4, Définition 2.4].

By Shapiro's lemma and the long exact sequence in Galois cohomology associated to the short exact sequence $0 \rightarrow \mathbb{Z} \rightarrow \mathbb{Z}\left[\Gamma_{k} / \Gamma_{L}\right] \rightarrow \widehat{T} \rightarrow 0$, the first term of (13) is isomorphic to $Q_{L} / \operatorname{Res}_{k / L}\left(Q_{k}\right)$ as above.

Furthermore, we have $\amalg_{\omega}^{2}(\widehat{T}) \cong H^{1}\left(k, \operatorname{Pic}\left(\bar{T}^{c}\right)\right)$ for all smooth compactifications $T^{c}$ of $T$ by [7, Proposition 9.5]. This gives the result.

In the following result, a more detailed version of Theorem 3 stated in the introduction, we determine the Brauer groups.

Theorem 4 Let $k$ be a field of characteristic zero with $H^{3}\left(k, \bar{k}^{\times}\right)=0$. Let $K / k$ be a field extension of degree 4 with Galois closure $K^{\mathrm{cl}}$. Let $P(t)$ be an irreducible quadratic polynomial over $k$ with splitting field L. Let $X^{c}$ be a smooth proper model of $X \subset \mathbb{A}_{k}^{5}$ defined by (1).

If we are in the case that $K / k$ is not Galois, that $P(t)$ remains irreducible over $K$ and that $K \cdot L / k$ is Galois with $\operatorname{Gal}(K \cdot L / L) \cong \mathbb{Z} / 4 \mathbb{Z}$, then we have $\operatorname{Br}\left(X^{c}\right) / \operatorname{Br}_{0}\left(X^{c}\right) \cong$ $\mathbb{Z} / 2 \mathbb{Z}$. Moreover, a representative for the non-trivial element of $\operatorname{Br}\left(X^{c}\right) / \operatorname{Br}_{0}\left(X^{c}\right)$ is 
given by $\operatorname{Cor}_{L / k}(t-\sqrt{a}, \chi)$, where $\chi \in \operatorname{Hom}(\operatorname{Gal}(K \cdot L / L), \mathbb{Q} / \mathbb{Z})$ is a character that is non-trivial in $Q_{L} / \operatorname{Res}_{k / L}\left(Q_{k}\right)$.

Otherwise, $\operatorname{Br}\left(X^{c}\right)=\operatorname{Br}_{0}\left(X^{c}\right)$.

Proof We have the following mutually disjoint cases, where we will first show that the Brauer group is trivial in cases (1)-(4); then we will show that it is non-trivial in case (5).

1. $P(t)$ is split over $K$, i.e. $L \subset K$,

2. $P(t)$ is irreducible over $K^{\mathrm{cl}}$,

3. $P(t)$ is irreducible over $K$ and split in $K^{\mathrm{cl}}$, with $K^{\mathrm{cl}} \neq K \cdot L$.

4. $P(t)$ is irreducible over $K$ and split in $K^{\mathrm{cl}}$, with $K^{\mathrm{cl}}=K \cdot L$ and $\operatorname{Gal}\left(K^{\mathrm{cl}} / L\right) \cong$ $\mathbb{Z} / 2 \mathbb{Z} \times \mathbb{Z} / 2 \mathbb{Z}$.

5. $P(t)$ is irreducible over $K$ and split in $K^{\mathrm{cl}}$, with $K^{\mathrm{cl}}=K \cdot L$ and $\operatorname{Gal}\left(K^{\mathrm{cl}} / L\right) \cong$ $\mathbb{Z} / 4 \mathbb{Z}$.

We can assume without loss of generality that $P(t)=c\left(t^{2}-a\right)$, where $a \in k^{\times}$is not a square and $c \in k^{\times}$.

Case (1). If the extension $K / k$ is Galois in this case, the statement follows from results in [25]: we refer to [25, Proposition $1.2(\mathrm{~d})]$ for the case $\operatorname{Gal}(K / k) \cong \mathbb{Z} / 4 \mathbb{Z}$ and to [25, Proposition 2.6] for the much harder case $\operatorname{Gal}(K / k) \cong \mathbb{Z} / 2 \mathbb{Z} \times \mathbb{Z} / 2 \mathbb{Z}$.

If $K / k$ is not Galois in case (1), then since $K \supset L=k(\sqrt{a})$, there exist $u, v \in k$ such that $K=L(\sqrt{u+v \sqrt{a}})$. The minimal polynomial of $\sqrt{u+v \sqrt{a}}$ over $k$ is $t^{4}-2 u t^{2}+u^{2}-a v^{2}$, with roots $\pm \sqrt{u \pm v \sqrt{a}}$ in $K^{\mathrm{cl}}$. Since $K / k$ is not Galois, $\sqrt{u-v \sqrt{a}}$ is not in $K$ and hence $d=u^{2}-a v^{2}$ is not a square in $k^{\times}$; in particular, $v \neq 0$. On the other hand, $K(\sqrt{d}) / k$ is Galois and hence it is the Galois closure of $K / k$. Since $\operatorname{Gal}(K(\sqrt{d}) / k)$ is a subgroup of $\mathcal{S}_{4}$ of order 8 , it must be the 2-Sylow subgroup $\mathcal{D}_{4}$ in $\mathcal{S}_{4}$. We now show that $H^{1}(k, \operatorname{Pic}(\bar{Y}))=0$.

The group $H^{1}\left(k, \operatorname{Pic}\left(\bar{T}^{c}\right)\right)$ (where again $\left.T=R_{K / k}^{1}\left(\mathbb{G}_{\mathrm{m}, K}\right)\right)$ is trivial, for example by [17, Proposition 1] (although full details are not given there: these can be found in unpublished work of Sansuc, see [20]). Since

$$
t^{4}-2 u t^{2}+u^{2}-a v^{2}=\left(t^{2}-(u+v \sqrt{a})\right)\left(t^{2}-(u-v \sqrt{a})\right)
$$

is a factorization into coprime polynomials over $L$, we have

$$
K \otimes_{k} L=L[t] /\left(\left(t^{2}-(u+v \sqrt{a})\right)\left(t^{2}-(u-v \sqrt{a})\right)\right)=K \oplus K^{\prime}
$$

with $K^{\prime}=L(\sqrt{u-v \sqrt{a}})$. Since $K \neq K^{\prime}$, we have $K \cap K^{\prime}=L$. Therefore, in Lemma 2,

$$
\begin{aligned}
Q_{L} & =\operatorname{ker}\left(H^{1}(L, \mathbb{Q} / \mathbb{Z}) \rightarrow H^{1}(K, \mathbb{Q} / \mathbb{Z}) \oplus H^{1}\left(K^{\prime}, \mathbb{Q} / \mathbb{Z}\right)\right) \\
& =\operatorname{ker}\left(H^{1}(L, \mathbb{Q} / \mathbb{Z}) \rightarrow H^{1}\left(K \cap K^{\prime}, \mathbb{Q} / \mathbb{Z}\right)\right)=0,
\end{aligned}
$$

so that $H^{1}(k, \operatorname{Pic}(\bar{Y}))=0$. Of course this implies $\operatorname{Br}(X)=\operatorname{Br}_{0}(X)$ by the short exact sequence (12). 
Case (2). In this case, $P(t)$ is irreducible over the Galois closure $K^{\mathrm{cl}}$ of the non-Galois extension $K / k$. The result then follows immediately from [25, Theorem 2.2].

Case (3). In this case, we know that $K^{\mathrm{cl}} \neq K \cdot L$ and that $P(t)$ is irreducible over $K$, but split over the Galois closure $K^{\mathrm{cl}}$, so $K \cdot L \subsetneq K^{\mathrm{cl}}$. Since $[K \cdot L: k]=8$, we have $\operatorname{Gal}\left(K^{\mathrm{cl}} / k\right) \cong \mathcal{S}_{4}$. Again by [17, Proposition 1], the group $H^{1}\left(k, \operatorname{Pic}\left(\bar{T}^{c}\right)\right)$ is trivial, so it is enough to prove the triviality of

$$
Q_{L}=\operatorname{ker}\left(H^{1}(L, \mathbb{Q} / \mathbb{Z}) \rightarrow H^{1}(K \cdot L, \mathbb{Q} / \mathbb{Z})\right)
$$

Non-triviality of this kernel would mean that there exists a non-trivial cyclic extension of $L$ contained in $K \cdot L$. But Gal $\left(K^{\mathrm{cl}} / L\right) \cong \mathcal{A}_{4}$ since it has index 2 in $\operatorname{Gal}\left(K^{\mathrm{cl}} / k\right) \cong \mathcal{S}_{4}$. Therefore, the Galois group of such an extension would be a normal subgroup of index 2 or 4 in $\mathcal{A}_{4}$, which does not exist. Therefore $Q_{L}=0$ and $\operatorname{Br}\left(X^{c}\right)=\operatorname{Br}_{0}\left(X^{c}\right)$.

Cases (4) and (5). In these cases, $K^{\mathrm{cl}}=K \cdot L$ has degree 8 over $k$. As in the part of case (1) where $K / k$ is not Galois, we have $\operatorname{Gal}\left(K^{\mathrm{cl}} / k\right) \cong \mathcal{D}_{4}$, with $K / k$ not Galois and $L \subset K^{\mathrm{cl}}$.

The group $\mathcal{D}_{4}$ has five subgroups of order 2, exactly one of which is normal; and it has three normal subgroups of order 4; moreover, any non-normal subgroup of order 2 is contained in exactly one normal subgroup of order 4 , the normal subgroup of order 2 is contained in all normal subgroups of order 4 . In the subfield lattice of $K^{\mathrm{cl}} / k$, we have the following intermediate fields, where we mark the normal extensions of $k$ by a box, and $K$ is any one of the non-normal extensions:

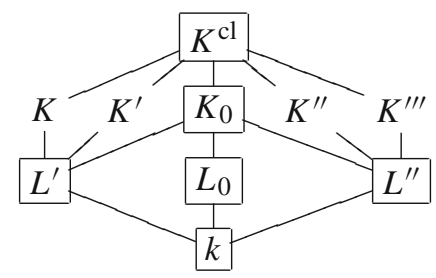

This diagram shows that $L=L^{\prime}$ in the non-Galois part of case (1), $L=L^{\prime \prime}$ in case (4) with $\operatorname{Gal}\left(K^{\mathrm{cl}} / L\right) \cong \mathbb{Z} / 2 \mathbb{Z} \times \mathbb{Z} / 2 \mathbb{Z}$, and $L=L_{0}$ in case (5) with $\operatorname{Gal}\left(K^{\mathrm{cl}} / L\right) \cong \mathbb{Z} / 4 \mathbb{Z}$. In all three cases, $\operatorname{Gal}\left(K^{\mathrm{cl}} / k\right) \cong \mathcal{D}_{4}$ implies that $H^{1}\left(k, \operatorname{Pic}\left(\bar{T}^{c}\right)\right)=0$, as we have seen in case (1).

In the two cases $L=L^{\prime \prime}$ and $L=L_{0}$, the polynomial $P(t)$ remains irreducible in $K$, and hence we have $K \otimes_{k} L \cong K(\sqrt{a})=K \cdot L=K^{\mathrm{cl}}$, so the group $Q_{L} \cong$ $H^{1}\left(K^{\mathrm{cl}} / L, \mathbb{Q} / \mathbb{Z}\right)$ has order 4 . Furthermore, $Q_{k}$ has order 2 because the diagram shows that there is only one non-trivial cyclic extension of $k$ contained in $K$, namely $L^{\prime}$. Since $L \cap K=k$, the restriction map $\operatorname{Res}_{k} / L$ sends $Q_{k}$ injectively into $Q_{L}$, and we conclude that $H^{1}(k, \operatorname{Pic}(\bar{Y})) \cong \mathbb{Z} / 2 \mathbb{Z}$. Now the short exact sequence (12) implies that the quotient $\operatorname{Br}\left(Y^{c}\right) / \operatorname{Br}_{0}\left(Y^{c}\right)$ - and therefore also $\operatorname{Br}\left(X^{c}\right) / \operatorname{Br}_{0}\left(X^{c}\right)$ - injects into $\mathbb{Z} / 2 \mathbb{Z}$.

By [4, Remark on p. 76], we know that $\operatorname{Br}(Y) / \operatorname{Br}_{0}(Y)$ is generated by the element $B=\operatorname{Cor}_{L / k}(t-\sqrt{a}, \chi) \in \operatorname{Br}(k(X))$, where $\chi \in Q_{L}$ with $\chi \notin Q_{k}$. 
Case (4). For $L=L^{\prime \prime}$, we will show that $B$ is ramified at $t=\infty$. This implies that $\operatorname{Br}\left(X^{c}\right)=\operatorname{Br}_{0}\left(X^{c}\right)$. In case (5) with $L=L_{0}$, we will show below that $B$ extends to a non-trivial element of $\operatorname{Br}\left(X^{c}\right)$.

We consider the quadratic extension $\left(K^{\mathrm{cl}}\right)^{\operatorname{ker}(\chi)}$ of $L$ associated to $\chi$. If this were $K_{0}$, then this subfield of $K^{\mathrm{cl}} / L$ would come from the subfield $L^{\prime}$ of $K / k$, so $\chi$ would be trivial in $Q_{L} / \operatorname{Res}_{k / L}\left(Q_{k}\right)$, contrary to our choice of $\chi$. So we may assume that $\left(K^{\mathrm{cl}}\right)^{\mathrm{ker}(\chi)}=K^{\prime \prime}$ in diagram $(14)$.

The variety $Y_{K^{\prime \prime}}=Y \times_{k} K^{\prime \prime}$ contains an open affine $K^{\prime \prime}$-subvariety $V$ defined by

$$
N_{K^{\mathrm{cl}} / K^{\prime \prime}}\left(\mathbf{z}_{1}\right) N_{K^{\mathrm{cl}} / K^{\prime \prime}}\left(\mathbf{z}_{2}\right)=c\left(t^{2}-a\right) \quad \text { and } \quad t \neq 0 .
$$

Let $W$ be the smooth affine $K^{\prime \prime}$-variety defined by

$$
N_{K / K^{\prime \prime}}\left(\mathbf{z}_{1}^{\prime}\right) N_{K / K^{\prime \prime}}\left(\mathbf{z}_{2}^{\prime}\right)=c\left(1-a t^{\prime 2}\right) .
$$

The open subvariety of $W$ defined by $t^{\prime} \neq 0$ is isomorphic to $V$ by the map $\left(t^{\prime}, \mathbf{z}_{1}^{\prime}, \mathbf{z}_{2}^{\prime}\right) \mapsto\left(1 / t^{\prime}, \mathbf{z}_{1}^{\prime} / t^{\prime}, \mathbf{z}_{2}^{\prime}\right)$. Let $D$ be the divisor of $W$ defined by $t^{\prime}=0$. It is easy to see that the divisor $D$ is geometrically irreducible. Hence $\bar{k} \cap K^{\prime \prime}(D)=K^{\prime \prime}$, where $K^{\prime \prime}(D)$ is the function field of $D$. The local ring $A_{D} \subset K^{\prime \prime}(Y)$ associated to the divisor $D$ is a discrete valuation ring and $\operatorname{ord}_{A_{D}}(t)=-1$. We have $\kappa_{A_{D}}=K^{\prime \prime}(D)$.

The natural restriction map $\operatorname{Res}_{k / K^{\prime \prime}}: \operatorname{Br}(Y) \rightarrow \operatorname{Br}\left(Y_{K^{\prime \prime}}\right)$ factorizes as $\operatorname{Res}_{k / K^{\prime \prime}}=$ $\operatorname{Res}_{L / K^{\prime \prime}} \circ \operatorname{Res}_{k / L}$. We will exchange restriction and corestriction using the double coset formula [18, Proposition I.1.5.6]. The coset decomposition is $\operatorname{Gal}(\bar{k} / k)=$ $\operatorname{Gal}(\bar{k} / L) \cup \sigma \operatorname{Gal}(\bar{k} / L)$ for some $\sigma \in \operatorname{Gal}(\bar{k} / k)$ that is non-trivial on $L$. Applying [10, Proposition 1.1.3] in the last step with $v_{A_{D}}(t \pm \sqrt{a})=-1$, we have

$$
\begin{aligned}
\partial_{A_{D}}\left(\operatorname{Res}_{k / K^{\prime \prime}}(B)\right) & =\partial_{A_{D}}\left(\operatorname{Res}_{L / K^{\prime \prime}}\left(\operatorname{Res}_{k / L}\left(\operatorname{Cor}_{L / k}(t-\sqrt{a}, \chi)\right)\right)\right) \\
& =\partial_{A_{D}}\left(\left(t-\sqrt{a}, \operatorname{Res}_{L / K^{\prime \prime}}(\chi)\right)+\left(t+\sqrt{a}, \operatorname{Res}_{L / K^{\prime \prime}}\left(\chi^{\sigma}\right)\right)\right) \\
& =-\operatorname{Res}_{L / \kappa_{A_{D}}}\left(\chi+\chi^{\sigma}\right) \in \operatorname{Hom}\left(\operatorname{Gal}\left(\bar{\kappa}_{A_{D}} / \kappa_{A_{D}}\right), \mathbb{Q} / \mathbb{Z}\right) .
\end{aligned}
$$

By diagram (14), $L^{\prime} \cdot K^{\prime \prime}=K^{\mathrm{cl}}$. Since $\chi$ corresponds to $K^{\prime \prime}$, we know that $\chi^{\sigma}$ corresponds to its conjugate $K^{\prime \prime \prime}$, hence $\chi+\chi^{\sigma}$ corresponds to $K_{0}$. Since

$$
\kappa_{A_{D}} \cap K_{0}=K^{\prime \prime}(D) \cap \bar{k} \cap K_{0}=K^{\prime \prime} \cap K_{0}=L,
$$

we have

$$
\operatorname{Res}_{L / \kappa_{A_{D}}}\left(\chi+\chi^{\sigma}\right) \neq 0 \in \operatorname{Hom}\left(\bar{\kappa}_{A_{D}} / \kappa_{A_{D}}, \mathbb{Q} / \mathbb{Z}\right) .
$$

Therefore, $\operatorname{Res}_{k / K^{\prime \prime}}(B) \notin \operatorname{Br}\left(Y_{K^{\prime \prime}}^{c}\right)$, hence $B \notin \operatorname{Br}\left(X^{c}\right)$.

Case (5). As discussed above in the context of case (4), it remains to show that $B=$ $\operatorname{Cor}_{L / k}(t-\sqrt{a}, \chi) \in \operatorname{Br}(k(X))$ extends to $\operatorname{Br}\left(X^{c}\right)$. We have $L=L_{0}$ in diagram (14).

It is sufficient to show that for any discrete valuation ring $A$ of $k(X)$ that corresponds to a valuation that is trivial on $k$, with residue field $\kappa_{A}$, the residue map $[10, \S 1.1]$ applied 
to $B$ gives the zero map

$$
\partial_{A}(B) \in H^{1}\left(\kappa_{A}, \mathbb{Q} / \mathbb{Z}\right)=\operatorname{Hom}\left(\operatorname{Gal}\left(\bar{\kappa}_{A} / \kappa_{A}\right), \mathbb{Q} / \mathbb{Z}\right) .
$$

Let us therefore focus on proving the triviality of $\partial_{A}(B)$ for any such discrete valuation ring $A$. Let $g \in \operatorname{Gal}\left(\bar{\kappa}_{A} / \kappa_{A}\right)$. We extend the embedding $k \subset \kappa_{A}$ to an embedding $\bar{k} \subset \bar{\kappa}_{A}$, so that $g$ acts also on $\bar{k}$. Let $K^{\mathrm{cl}, g}$ be the subfield of $K^{\mathrm{cl}}$ fixed by $g$, with cyclic Galois group $\operatorname{Gal}\left(K^{\mathrm{cl}} / K^{\mathrm{cl}, g}\right)$.

Since $X$ is geometrically integral, $X \times_{k} K^{\mathrm{cl}}$ is an irreducible variety with function field $K^{\mathrm{cl}}(X)$. We can extend $A$ to a discrete valuation ring $A_{K^{\mathrm{cl}}}$ of $K^{\mathrm{cl}}(X)$ with residue field $\kappa_{A_{K} \mathrm{cl}}=\kappa_{A} \cdot K^{\mathrm{cl}}$. Indeed, the completion of $k(X)$ for the given valuation is isomorphic to $\kappa_{A}((T))$, where $T$ is a uniformizer. The map $k(X) \rightarrow \kappa_{A}((T))$ gives the natural map

$$
K^{\mathrm{cl}} \otimes_{k} k(X) \rightarrow K^{\mathrm{cl}} \otimes_{k} \kappa_{A}((T))
$$

Composing with the natural map $K^{\mathrm{cl}} \otimes_{k} \kappa_{A}((T)) \rightarrow\left(K^{\mathrm{cl}} \cdot \kappa_{A}\right)((T))$, we have the map

$$
K^{\mathrm{cl}} \otimes_{k} k(X) \rightarrow\left(K^{\mathrm{cl}} \cdot \kappa_{A}\right)((T)) .
$$

Using $K^{\mathrm{cl}}(X)=K^{\mathrm{cl}} \otimes_{k} k(X)$, we get the map

$$
K^{\mathrm{cl}}(X) \rightarrow\left(K^{\mathrm{cl}} \cdot \kappa_{A}\right)((T))
$$

that is injective since both objects are fields. We can see that $\left(K^{\mathrm{cl}} \cdot \kappa_{A}\right)((T))$ is a discrete valuation field and the valuation is given by the uniformizer $T$; the valuation restricted to $K^{\mathrm{cl}}(X)$ induces a discrete valuation of $K^{\mathrm{cl}}(X)$, and we denote the respective discrete valuation ring by $A_{K^{\mathrm{cl}}}$ and the residue field by $\kappa_{A_{K^{\mathrm{cl}}}}$. We have the induced map $A_{K^{\mathrm{cl}}} \rightarrow\left(K^{\mathrm{cl}} \cdot \kappa_{A}\right)[[T]]$. Then we have the injection $\kappa_{A_{K^{\mathrm{cl}}}} \rightarrow K^{\mathrm{cl}} \cdot \kappa_{A}$, since the residue field of $\left(K^{\mathrm{cl}} \cdot \kappa_{A}\right)[[T]]$ is $K^{\mathrm{cl}} \cdot \kappa_{A}$. Since $k(X)$ is dense in $\kappa_{A}((T))$, we conclude that $K^{\mathrm{cl}}(X)$ is dense in $\left(K^{\mathrm{cl}} \cdot \kappa_{A}\right)((T))$, which implies that the map $\kappa_{A_{K} \mathrm{cl}} \rightarrow K^{\mathrm{cl}} \cdot \kappa_{A}$ is surjective. Therefore the map $\kappa_{A_{K^{\mathrm{cl}}}} \rightarrow K^{\mathrm{cl}} \cdot \kappa_{A}$ is an isomorphism.

For any intermediate field $M$ of $K^{\mathrm{cl}} / k$, we have similarly the valuation ring $A_{M}$ of $M(X)$ with residue field $\kappa_{A_{M}}$. We write $A_{g}$ for $A_{K^{\mathrm{cl}, g}}$.

To show that $\partial_{A}(B)(g)$ is zero for any $g$, we distinguish two cases:

(5.i) $L \not \subset K^{\mathrm{cl}, g}$, (5.ii) $L \subset K^{\mathrm{cl}, g}$.

Case (5.i). We assume that $L \not \subset K^{\mathrm{cl}, g}$. We note that $\mathrm{Gal}\left(K^{\mathrm{cl}} / K^{\mathrm{cl}, g}\right)$ is a cyclic subgroup of $\mathcal{D}_{4}$. We see in diagram (14) that the only such subfields of $K^{\mathrm{cl}}$ not containing $L=L_{0}$ are $K, K^{\prime}, K^{\prime \prime}, K^{\prime \prime \prime}$. In any case, $L \cdot K^{\mathrm{cl}, g}=K^{\mathrm{cl}}$. 
By [10, Proposition 1.1.1], we have the commutative diagram

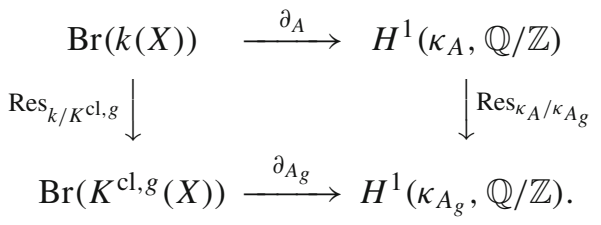

Since $\kappa_{A_{g}}=\kappa_{A} \cdot K^{\mathrm{cl}, g}$, we have $g \in \operatorname{Gal}\left(\bar{\kappa}_{A} / \kappa_{A_{g}}\right)$. Hence

$$
\partial_{A}(B)(g)=\operatorname{Res}_{\kappa_{A} / \kappa_{A g}}\left(\partial_{A}(B)\right)(g)=\partial_{A_{g}}\left(\operatorname{Res}_{k / K^{\mathrm{cl}, g}}(B)\right)(g) .
$$

By definition, we have $\operatorname{Res}_{k / K^{\mathrm{cl}, g}}(B)=\operatorname{Res}_{k / K^{\mathrm{cl}, g}}\left(\operatorname{Cor}_{L / k}(t-\sqrt{a}, \chi)\right)$. Since $L \cap K^{\mathrm{cl}, g}=k$, we have $\operatorname{Gal}(\bar{k} / k)=\operatorname{Gal}(\bar{k} / L) \cdot \operatorname{Gal}\left(\bar{k} / K^{\mathrm{cl}, g}\right)$. Exchanging restriction and corestriction using [18, Proposition I.1.5.6] gives

$$
\operatorname{Res}_{k / K^{\mathrm{cl}, g}}(B)=\operatorname{Cor}_{L \cdot K^{\mathrm{cl}, g} / K^{\mathrm{cl}, g}}\left(\operatorname{Res}_{L / L \cdot K^{\mathrm{cl}, g}}(t-\sqrt{a}, \chi)\right) .
$$

Since $L \cdot K^{\mathrm{cl}, g}=K^{\mathrm{cl}}$, the projection formula for cup products gives

$$
\operatorname{Res}_{L / L \cdot K^{\mathrm{cl}, g}}(t-\sqrt{a}, \chi)=\left(t-\sqrt{a}, \operatorname{Res}_{L / K^{\mathrm{cl}}}(\chi)\right) .
$$

But $\operatorname{Res}_{L / K^{\mathrm{cl}}}(\chi)=0$ by definition of $Q_{L}$, hence $\operatorname{Res}_{k / K^{\mathrm{cl}, g}}(B)=0$.

Case (5.ii). We assume that $L \subset K^{\mathrm{cl}, g}$. We work on $X_{L}=X \times_{k} L$. There are maps $X_{K^{\mathrm{cl}}} \rightarrow X_{L} \rightarrow X$. Let $A_{L}=A_{g} \cap L(X)$ be the corresponding discrete valuation ring on $L(X)$. Using the diagram

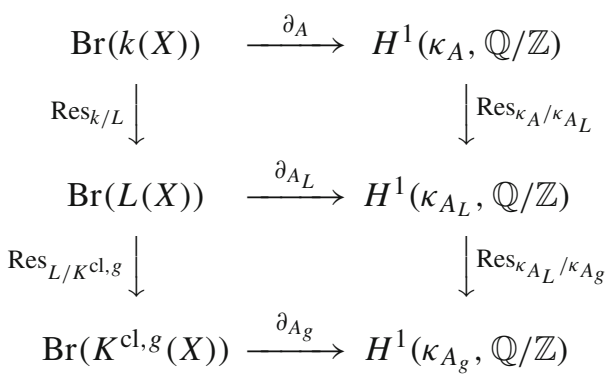

we get the equality

$$
\partial_{A_{g}}\left(\operatorname{Res}_{k / K^{\mathrm{cl}, g}}(B)\right)=\operatorname{Res}_{\kappa_{A_{L}} / \kappa_{A g}}\left(\partial_{A_{L}}\left(\operatorname{Res}_{k / L}(B)\right)\right) .
$$

Thus it suffices to show that $\partial_{A_{L}}\left(\operatorname{Res}_{k / L}(B)\right)=0$ (independent of $g$ ).

We apply the double coset formula again: in this case, the coset decomposition reduces to $\operatorname{Gal}(\bar{k} / k)=\operatorname{Gal}(\bar{k} / L) \cup \sigma \operatorname{Gal}(\bar{k} / L)$, for any $\sigma \in \operatorname{Gal}(\bar{k} / k)$ that is nontrivial on $L$. Therefore, 


$$
\operatorname{Res}_{k / L}(B)=(t-\sqrt{a}, \chi)+\left(t+\sqrt{a}, \chi^{\sigma}\right),
$$

where $\chi^{\sigma}$ is obtained from $\chi$ via conjugation with $\sigma$. We note that $X_{L}$ is given by $c(t-\sqrt{a})(t+\sqrt{a})=N_{K^{\mathrm{cl}} / L}(\mathbf{z})$ since $K^{\mathrm{cl}}=K \otimes_{k} L$. By [10, Proposition 1.1.3], we have

$$
\partial_{A_{L}}\left(\operatorname{Res}_{k / L}(B)\right)=v_{A_{L}}(t-\sqrt{a}) \operatorname{Res}_{L / \kappa_{A_{L}}}(\chi)+v_{A_{L}}(t+\sqrt{a}) \operatorname{Res}_{L / \kappa_{A_{L}}}\left(\chi^{\sigma}\right) .
$$

If $v_{A_{L}}(t-\sqrt{a})=v_{A_{L}}(t+\sqrt{a})=0$, then (15) vanishes.

If $v_{A_{L}}(t-\sqrt{a})>0$, then $v_{A_{L}}(t+\sqrt{a})=0$ since $v_{A_{L}}(2 \sqrt{a})=0$. Therefore,

$$
v_{A_{L}}(t-\sqrt{a})=v_{A_{L}}(t-\sqrt{a})+v_{A_{L}}(t+\sqrt{a})=v_{A_{L}}\left(N_{K^{\mathrm{cl}} / L}(\mathbf{z})\right)-v_{A_{L}}(c) .
$$

Now $v_{A_{L}}(c)=0$. Let $M=\kappa_{A_{L}} \cap K^{\mathrm{cl}}$. Then

$$
K^{\mathrm{cl}} \otimes_{L} M \cong \bigoplus_{i=1}^{[M: L]} K^{\mathrm{cl}}
$$

since $K^{\mathrm{cl}} / L$ is Galois and $M$ is totally split in $K^{\mathrm{cl}}$. Since $A_{L}$ is a discrete valuation ring of $L(X)$, we have $\widehat{L(X)} \cong \kappa_{A_{L}}((T))$, where $T$ is a uniformizer. Hence

$$
\widehat{L(X)} \cap K^{\mathrm{cl}}=\kappa_{A_{L}} \cap K^{\mathrm{cl}}=M .
$$

We have

$$
K^{\mathrm{cl}} \otimes_{L} \widehat{L(X)} \cong K^{\mathrm{cl}} \otimes_{L} M \otimes_{M} \widehat{L(X)} \cong \bigoplus_{i=1}^{[M: L]} K^{\mathrm{cl}} \otimes_{M} \widehat{L(X)}
$$

Therefore,

$$
\begin{aligned}
v_{A_{L}}\left(N_{K^{\mathrm{cl}} / L}(\mathbf{z})\right) & =v_{A_{L}}\left(N_{K^{\mathrm{cl}} \otimes_{L}} \widehat{L(X)} / \widehat{L(X)}(\mathbf{z})\right) \\
& =\sum_{i=1}^{[M: L]} v_{A_{L}}\left(N_{K^{\mathrm{cl}} \otimes_{M} \widehat{L(X)} / \widehat{L(X)}}\left(\mathbf{z}_{i}\right)\right) \\
& =\sum_{i=1}^{[M: L]}\left[K^{\mathrm{cl}} \otimes_{M} \widehat{L(X)}: \widehat{L(X)}\right] \cdot v_{A_{K}^{\mathrm{cl}}}\left(\mathbf{z}_{i}\right) .
\end{aligned}
$$

This is a multiple of $\left[K^{\mathrm{cl}}: M\right]$. Indeed, since $\widehat{L(X)} \cap K^{\mathrm{cl}}=M$, we know that $K^{\mathrm{cl}} \otimes_{M} \widehat{L(X)}$ is an unramified field extension over $\widehat{L(X)}$ of degree

$$
\left[K^{\mathrm{cl}} \otimes_{M} \widehat{L(X)}: \widehat{L(X)}\right]=\left[K^{\mathrm{cl}}: M\right],
$$


and $v_{A_{K^{\mathrm{cl}}}}$ has values in $\mathbb{Z}$. Since the order of $\operatorname{Res}_{L / \kappa_{A}}(\chi)$ divides $\left[K^{\mathrm{cl}}: M\right],(15)$ vanishes. The case $v_{A_{L}}(t+\sqrt{a})>0$ is analogous.

If $v_{A_{L}}(t-\sqrt{a})<0$, then $v_{A_{L}}(t+\sqrt{a})=v_{A_{L}}(t-\sqrt{a})$ since $v_{A_{L}}(2 \sqrt{a})=0$. Since $\operatorname{Gal}\left(K^{\mathrm{cl}} / L\right) \cong \mathbb{Z} / 4 \mathbb{Z}$, it is easy to see that $\chi^{\sigma}=-\chi$. Hence (15) vanishes. This completes the proof of $\partial_{A_{L}}\left(\operatorname{Res}_{k / L}(B)\right)=0$ in case (5.ii), and therefore the proof of the non-triviality of $\operatorname{Br}\left(X^{c}\right)$ in case (5).

Corollary 3 Let $K / k$ be a quartic extension of number fields with $k=\mathbb{Q}$. Let $P(t) \in$ $\mathbb{Q}[t]$ be an irreducible quadratic polynomial with splitting field $L$.

If we are not in the case where $K / k$ is not Galois, $P(t)$ is irreducible over $K$ and $K \cdot L / k$ is Galois with $\operatorname{Gal}(K \cdot L / L) \cong \mathbb{Z} / 4 \mathbb{Z}$, then the Hasse principle and weak approximation hold on $X \subset \mathbb{A}_{\mathbb{Q}}^{5}$ defined by equation (1)

Remark 3 J.-L. Colliot-Thélène suggested an alternative method to prove Theorem 4 in case (1), which we will sketch here. The proof of Theorem 1 shows that $X^{c}$ is birational to a smooth, projective $k$-variety $U^{c}$ equipped with a morphism $p: U^{c} \rightarrow S^{c}$ to a smooth, projective conic $S^{c}$ over $k$, in such a way that the generic fibre of $p$ is a smooth quadric $U_{k(S)}^{c}$ in $\mathbb{P}_{k(S)}^{4}$. One can then check that $\operatorname{Br}\left(U_{k(S)}^{c}\right) \cong \operatorname{Br}(k(S))$ and that $\operatorname{Br}(k)$ surjects onto $\operatorname{Br}\left(S^{c}\right)$. An explicit calculation with residues of elements of $\operatorname{Br}\left(U_{k(S)}^{c}\right)$ then allows one to conclude that $\operatorname{Br}(k)$ surjects onto $\operatorname{Br}\left(U^{c}\right)$.

\section{Failure of weak approximation}

Finally, we give a concrete example of a Brauer-Manin obstruction to weak approximation caused by the non-trivial element of $\operatorname{Br}\left(X^{c}\right) / \operatorname{Br}_{0}\left(X^{c}\right)$ described in Theorem 4 .

Example 1 Let $K=\mathbb{Q}(\sqrt[4]{17})$ and let $P(t)=t^{2}+1$. Let $X \subset \mathbb{A}_{\mathbb{Q}}^{5}$ be defined by $t^{2}+1=N_{K / \mathbb{Q}}(\mathbf{z})$. Then weak approximation does not hold on $X$.

Indeed, consider the adelic point $\left(\mathbf{x}_{v}\right) \in \prod_{v} X\left(k_{v}\right)$ given by

$$
\mathbf{x}_{v}=\left(t_{v}, \mathbf{z}_{v}\right)= \begin{cases}(0,1), & v \neq 17 \\ \left(7, \mathbf{z}_{17}\right), & v=17\end{cases}
$$

where $\mathbf{z}_{17} \in \mathbb{Q}_{17}(\sqrt[4]{17})$ is a solution of $7^{2}+1=N_{\mathbb{Q}_{17}(\sqrt[4]{17}) / \mathbb{Q}_{17}}\left(\mathbf{z}_{17}\right)$. Such a solution exists: 50 is even a fourth power in $\mathbb{Q}_{17}$ by Hensel's lemma, since $50 \equiv 2^{4}(\bmod 17)$.

Let $\chi$ be a primitive character of the cyclic group $\operatorname{Gal}(K(i) / \mathbb{Q}(i)) \cong \mathbb{Z} / 4 \mathbb{Z}$, regarded as element of $H^{2}(\mathbb{Q}(i), \mathbb{Z})$. Let $B=\operatorname{Cor}_{\mathbb{Q}(i) / \mathbb{Q}}(t-i, \chi)$. By Theorem 4 , this defines a non-trivial element in $\operatorname{Br}\left(X^{c}\right)$. Since

$$
\sum_{v \in \Omega_{\mathbb{Q}}} \operatorname{inv}_{v}(B((0,1)))=0 \in \mathbb{Q} / \mathbb{Z}
$$

it suffices to check that $\operatorname{inv}_{17}\left(B\left(\mathbf{x}_{17}\right)\right) \neq \operatorname{inv}_{17}(B((0,1)))$. To do this concrete calculation, one needs to fix an embedding of $\mathbb{Q}(i)$ into $\mathbb{Q}_{17}$. Let $\alpha \in \mathbb{Q}_{17}$ be such that 
$\alpha^{2}+1=0$ and $\alpha \equiv 4(\bmod 17)$. Consider the embedding $\iota_{\alpha}: \mathbb{Q}(i) \rightarrow \mathbb{Q}_{17}$ given by $i \mapsto \alpha$. By functoriality, this gives an element $\chi_{\alpha} \in H^{2}\left(\mathbb{Q}_{17}, \mathbb{Z}\right)$. The cup products

$$
\mathbb{Q}(i)^{*} \times H^{2}(\mathbb{Q}(i), \mathbb{Z}) \rightarrow \operatorname{Br}(\mathbb{Q}(i))
$$

and

$$
\mathbb{Q}_{17}^{*} \times H^{2}\left(\mathbb{Q}_{17}, \mathbb{Z}\right) \rightarrow \operatorname{Br}\left(\mathbb{Q}_{17}\right)
$$

are compatible with the functorial maps induced by $\iota_{\alpha}$. If $\chi_{-\alpha}$ is the image of $\chi$ via the embedding $\mathbb{Q}(i) \rightarrow \mathbb{Q}_{17}$ defined by $i \mapsto-\alpha$, then one easily checks that $\chi_{-\alpha}=-\chi_{\alpha} \in H^{2}\left(\mathbb{Q}_{17}, \mathbb{Z}\right)$. Hence we get the equalities

$$
\begin{aligned}
B & \left(\mathbf{x}_{17}\right)-B((0,1)) \\
& =\operatorname{Res}_{\mathbb{Q} / \mathbb{Q}_{17}}\left(\operatorname{Cor}_{\mathbb{Q}(i) / \mathbb{Q}}(t-i, \chi)\right)\left(\mathbf{x}_{17}\right)-\operatorname{Res}_{\mathbb{Q} / \mathbb{Q}_{17}}\left(\operatorname{Cor}_{\mathbb{Q}}(i) / \mathbb{Q}\right. \\
& =(7-i, \chi))((0,1)) \\
& =\left(7-\alpha, \chi_{\alpha}\right)+\left(7+\alpha,-\chi_{\alpha}\right)-\left(\left(-\alpha, \chi_{\alpha}\right)-\left(\alpha,-\chi_{\alpha}\right)\right. \\
& =\left(\frac{7-\alpha}{7+\alpha}, \chi_{\alpha}\right)-\left(-1, \chi_{\alpha}\right) \\
& =\left((7-\alpha)^{2}, \chi_{\alpha}\right)
\end{aligned}
$$

in which we used the double coset formula and the bilinearity of the cup product, together with the fact that $(7-\alpha)(7+\alpha)=50$ and -1 are fourth powers in $\mathbb{Q}_{17}$. Now we only need to check that $(7-\alpha)^{2}$ is not a norm for the extension $\mathbb{Q}_{17}(\sqrt[4]{17}) / \mathbb{Q}_{17}$, but this is clear since $(7-4)^{2}=9$ is not a fourth power modulo 17 .

Acknowledgments The first named author was supported by Grant DE 1646/2-1 of the Deutsche Forschungsgemeinschaft and grant 200021_124737/1 of the Schweizer Nationalfonds. The second named author was supported by a PhD fellowship of the Research Foundation-Flanders (FWO). The third named author was supported by National Key Basic Research Program of China (Grant No. 2013CB834202) and National Natural Science Foundation of China (Grant Nos. 11371210 and 11321101). This collaboration was supported by the Center for Advanced Studies of LMU München. We thank T. D. Browning, J.-L. Colliot-Thélène, C. Demarche and B. Kunyavskiı̌ for useful discussions and remarks. Finally, we thank the referee for his suggestions for improvement.

\section{References}

1. Browning, T.D., Heath-Brown, D.R.: Quadratic polynomials represented by norm forms. Geom. Funct. Anal. 22(5), 1124-1190 (2012)

2. Bourbaki, N.: Éléments de mathématique. 23. Première partie: Les structures fondamentales de l'analyse. Livre II: Algèbre. Chapitre 8: Modules et Anneaux Semi-Simples. Actualités Sci. Ind., vol. 1261. Hermann, Paris (1958)

3. Colliot-Thélène, J.-L.: Points rationnels sur les fibrations. In: Higher Dimensional Varieties and Rational Points (Budapest, 2001). Bolyai Soc. Math. Stud., vol. 12, pp. 171-221. Springer, Berlin (2003)

4. Colliot-Thélène, J.-L., Harari, D., Skorobogatov, A. N.: Valeurs d'un polynôme à une variable représentées par une norm. In: Number Theory and Algebraic Geometry. London Math. Soc. Lecture Note Ser., vol. 303, pp. 69-89. Cambridge Univ. Press, Cambridge (2003) 
5. Colliot-Thélène, J.-L., Salberger, P.: Arithmetic on some singular cubic hypersurfaces. Proc. London Math. Soc. (3) 58(3), 519-549 (1989)

6. Colliot-Thélène, J.-L., Sansuc, J.J.: La descente sur les variétés rationnelles. II. Duke Math. J. 54(2), 375-492 (1987)

7. Colliot-Thélène, J.-L., Sansuc, J.J.: Principal homogeneous spaces under flasque tori: applications. J. Algebra 106(1), 148-205 (1987)

8. Colliot-Thélène, J.-L., Sansuc, J.-J., Swinnerton-Dyer, P.: Intersections of two quadrics and Châtelet surfaces. I. J. Reine Angew. Math. 373, 37-107 (1987)

9. Colliot-Thélène, J.-L., Sansuc, J.-J., Swinnerton-Dyer, P.: Intersections of two quadrics and Châtelet surfaces. II. J. Reine Angew. Math. 374, 72-168 (1987)

10. Colliot-Thélène, J.-L., Swinnerton-Dyer, P.: Hasse principle and weak approximation for pencils of Severi-Brauer and similar varieties. J. Reine Angew. Math. 453, 49-112 (1994)

11. Colliot-Thélène, J.-L., Skorobogatov, A.N.: Descent on fibrations over $\mathbf{P}_{k}^{1}$ revisited. Math. Proc. Cambridge Philos. Soc. 128(3), 383-393 (2000)

12. Colliot-Thélène, J.-L., Skorobogatov, A.N., Swinnerton-Dyer, P.: Rational points and zero-cycles on fibred varieties: Schinzel's hypothesis and Salberger's device. J. Reine Angew. Math. 495, 1-28 (1998)

13. Fouvry, E., Iwaniec, H.: Gaussian primes. Acta Arith. 79(3), 249-287 (1997)

14. Harari, D.: Flèches de spécialisations en cohomologie étale et applications arithmétiques. Bull. Soc. Math. France 125(2), 143-166 (1997)

15. Hasse, H.: Die Normenresttheorie relativ-Abelscher Zahlkörper als Klassenkörpertheorie im Kleinen. J. Reine Angew. Math. 162, 145-154 (1930)

16. Heath-Brown, D.R., Skorobogatov, A.N.: Rational solutions of certain equations involving norms. Acta Math. 189(2), 161-177 (2002)

17. Kunyavskiı̌, B.È.: Arithmetic properties of three-dimensional algebraic tori. Zap. Nauchn. Sem. Leningrad. Otdel. Mat. Inst. Steklov. (LOMI) 116, 102-107 (1982)

18. Neukirch, J., Schmidt, A., Wingberg, K.: Cohomology of Number Fields. Grundlehren der Mathematischen Wissenschaften, vol. 323, 2nd edn. Springer, Berlin (2008)

19. Sansuc, J.-J.: Groupe de Brauer et arithmétique des groupes algébriques linéaires sur un corps de nombres. J. Reine Angew. Math. 327, 12-80 (1981)

20. Sansuc, J.-J.: Le principe de Hasse normique dans le cas diédral non galoisien. Unpublished (1981)

21. Jones, M.S.: A note on a theorem of Heath-Brown and Skorobogatov. Q. J. Math. 64(4), 1239-1251 (2013)

22. Skorobogatov, A.N.: Beyond the Manin obstruction. Invent. Math. 135(2), 399-424 (1999)

23. Skorobogatov, A.N.: Torsors and Rational Points. Cambridge Tracts in Mathematics, vol. 144. Cambridge University Press, Cambridge (2001)

24. Schindler, D., Skorobogatov, A.N.: Norms as products of linear polynomials. J. Lond. Math. Soc. (2) 89(2), 559-580 (2014)

25. Wei, D.: On the equation $N_{K / k}(\Xi)=P(t)$. Proc. Lond. Math. Soc. (2014). doi:10.1112/plms/pdu035 